\title{
Diabetes Evaluation Sheet
}

\author{
Hisayuki Katsuyama ${ }^{\mathrm{a}}$, Hiroki Adachi ${ }^{\mathrm{a}}$, Yoshinori Masui ${ }^{\mathrm{a}}$, Mariko Hakoshima ${ }^{\mathrm{a}}$, \\ Yoko Waragai ${ }^{\mathrm{a}}$, Tadanao Harigae ${ }^{\mathrm{a}}$, Yusuke Kondo ${ }^{\mathrm{a}}$, \\ Takehiro Iijima $^{\text {a }}$, Hidekatsu Yanai ${ }^{a}$, b
}

Our institute, National Center for Global Health and Medicine Kohnodai Hospital treats many severe diabetic patients and studies various aspects of diabetes. To improve the quality of diagnosis and treatment for diabetes and also to accelerate studies on diabetes worldwide, here we will show our "NCGM Kohnodai Diabetes Evaluation Sheet" (Fig. 1).

This sheet includes the information about age, sex, body height and weight, body mass index, classification (etiology) of diabetes, duration of diabetes, treatments for diabetes, glycemic control, macrovascular and microvascular complications, insulin secretary capacity and insulin resistance, and coronary risk factors. In this sheet, we evaluate nephropathy according to the classification of diabetic nephropathy (The Japan Diabetes Society) made based on the study by Wada $\mathrm{T}$ et al [1], and we also evaluate estimated glomerular filtration rate (eGFR) category in chronic kidney disease (CKD) by using KDIGO 2012 CKD Guideline [2]. Furthermore, we evaluate insulin resistance by using homeostasis model assessment of insulin resistance (HOMA-IR), and also evaluate intrinsic insulin secretary capacity by using homeostasis model assessment of $\beta$-cell function (HOMA- $\beta$ ) and C-peptide immunoreactivity index (CPI index) $[3,4]$.

Therefore, NCGM Kohnodai Diabetes Evaluation Sheet helps us to make a valid diagnosis and an appropriate treatment for severe diabetic patients. This sheet is also very useful for educating young doctors about diabetes. We produce two to three diabetes specialists every year. Furthermore, this sheet may contribute to our research activities. We have reported 83 English original articles (total impact factor, 185.2) since 2013, by using this sheet.

We strongly hope that the diagnosis and treatment for dia- betes, and research of diabetes worldwide will develop by using this sheet.

\section{Conflict of Interest}

The authors declare that they have no conflict of interest concerning this article.

\section{References}

1. Wada T, Haneda M, Furuichi K, Babazono T, Yokoyama $\mathrm{H}$, Iseki K, Araki S, et al. Clinical impact of albuminuria and glomerular filtration rate on renal and cardiovascular events, and all-cause mortality in Japanese patients with type 2 diabetes. Clin Exp Nephrol. 2014;18(4):613-620.

2. Kidney Disease: Improving Global Outcomes (KDIGO) CKD Work Group. KDIGO 2012 clinical practice guideline for the evaluation and management of chronic kidney disease. Kidney Int Suppl. 2013:3;1-150.

3. Matthews DR, Hosker JP, Rudenski AS, Naylor BA, Treacher DF, Turner RC. Homeostasis model assessment: insulin resistance and beta-cell function from fasting plasma glucose and insulin concentrations in man. Diabetologia. 1985;28(7):412-419.

4. Iwata M, Matsushita Y, Fukuda K, Wakura T, Okabe K, Koshimizu Y, Fukushima Y, et al. Secretory units of islets in transplantation index is a useful predictor of insulin requirement in Japanese type 2 diabetic patients. J Diabetes Investig. 2014;5(5):570-580.

Manuscript submitted May 22, 2018, accepted June 18, 2018

a Department of Internal Medicine, National Center for Global Health and Medicine Kohnodai Hospital, Chiba, Japan

${ }^{\mathrm{b}}$ Corresponding Author: Hidekatsu Yanai, Department of Internal Medicine, National Center for Global Health and Medicine Kohnodai Hospital, 1-7-1 Kohnodai, Ichikawa, Chiba, 272-8516, Japan.

Email: dyanai@hospk.ncgm.go.jp

doi: https://doi.org/10.14740/jem513w 


\section{NCGM Kohnodai Diabetes Evaluation Sheet}

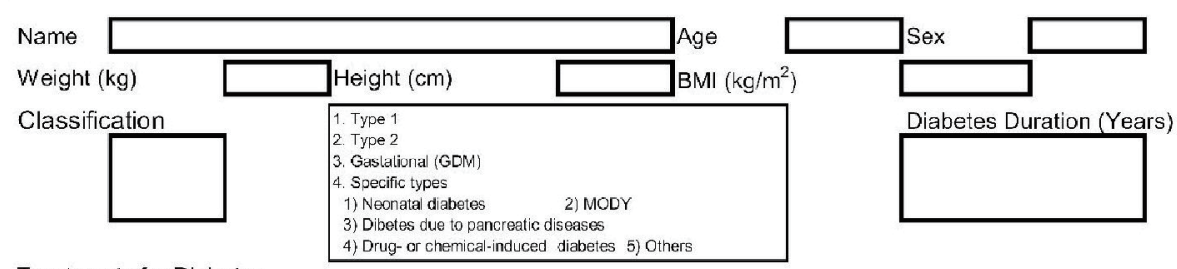

Treatments for Diabetes

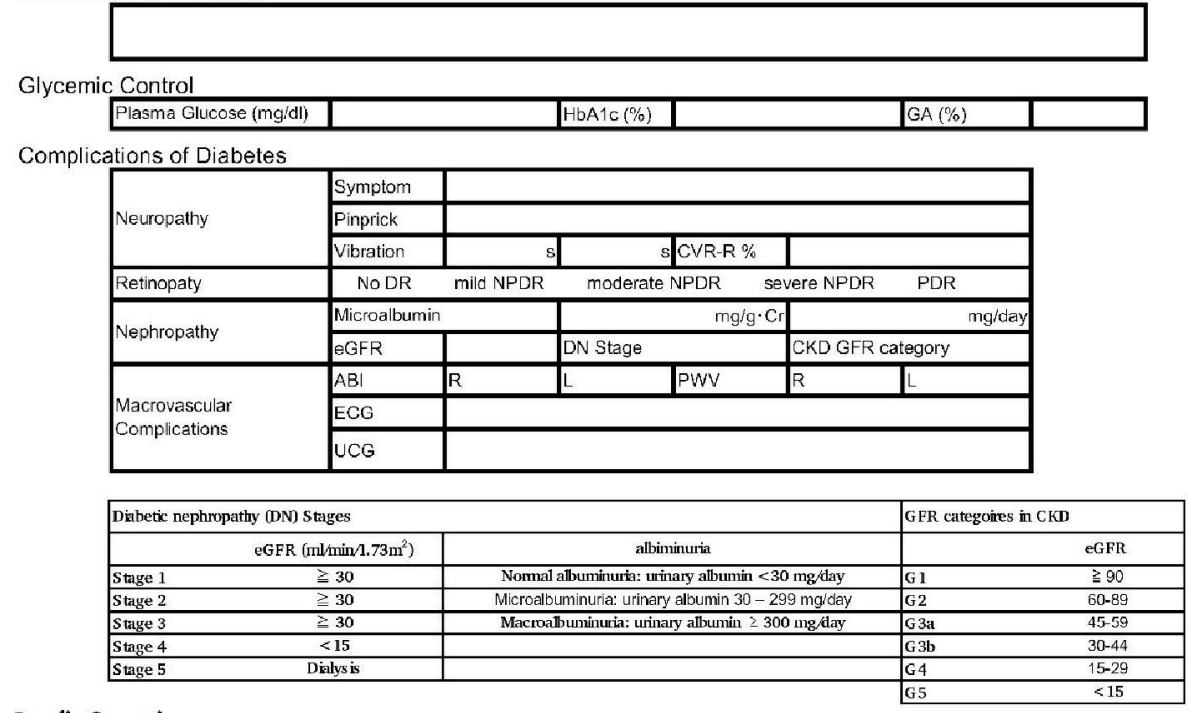

Insulin S ecretion

\begin{tabular}{|c|c|c|}
\hline & Fasting & (2-hour after meal) or (6-min after glucagon bad) \\
\hline \multicolumn{3}{|c|}{ Phasma G lucose (mg/dl) } \\
\hline \multicolumn{3}{|c|}{ Plasma Insulin ( $\mu \mid \cup / I)$} \\
\hline \multicolumn{3}{|c|}{ Plasma C-peptide (ng/nl) } \\
\hline \multicolumn{3}{|c|}{ Urine C-peptide $(\mu \mathrm{g} / \mathrm{day})$} \\
\hline \multicolumn{3}{|r|}{ Insulin-dependance } \\
\hline \multicolumn{3}{|l|}{ HOMA-3 } \\
\hline \multicolumn{3}{|l|}{ CPR index } \\
\hline $\begin{array}{l}\text { HOMA-IR }=\mathbf{f} \\
\text { HOMA- } \beta=36 \\
\text { CPR Index }=\mathbf{1}\end{array}$ & $\begin{array}{l}\text { (e 2.5: in } \\
3 \text { i) } 24 \\
\text { ie }[<1 .\end{array}$ & \\
\hline
\end{tabular}

\begin{tabular}{|c|c|c|c|c|}
\hline & Fasting CPR & CPR after glucagon load & $\triangle \mathrm{CPR}$ (after glucagon load) & Urinary CPR \\
\hline Insulin dependent & $\leqq 0.5 \mathrm{ng} / \mathrm{ml}$ & $\leqq 1.0 \mathrm{ng} / \mathrm{ml}$ & $\leqq 0.5 \mathrm{ng} / \mathrm{ml}$ & $\leqq 20 \mu \mathrm{g}^{\prime} \mathrm{day}$ \\
\hline Insullin non-dependent & $31.0 \mathrm{ng} / \mathrm{ml}$ & $\geqq 2.0 \mathrm{ng} / \mathrm{ml}$ & & $\geqq 30 \mu g^{\prime} d a y$ \\
\hline prefer to ius ulin therapy & & $51.8 \mathrm{ng} / \mathrm{ml}$ & $\leq 0.7 \mathrm{ng} / \mathrm{ml}$ & $\leqq 30 \mu \mathrm{g} / \mathrm{day}$ \\
\hline
\end{tabular}

Coronary Risk Factors

\begin{tabular}{|l|l|}
\hline B lood pressure (mmHg) & \\
\hline LDL-C (mig/di) & \\
\hline HDL-C (mg/d) & \\
\hline TG (mg/dl) & \\
\hline S moking (pack-years) & \\
\hline
\end{tabular}

Desidered values
- Blood press ure (for diabetic patients)
$<130,80$
- LDL-G $<120$ mg, $/ 11$
( $<100$; secondary prevention)
- HDL $>40 \mathrm{mg} / \mathrm{dl}$
$-\mathrm{TG}<150 \mathrm{mg} / \mathrm{dl}$

ARI, anlle brachisl press tre index; CKD, chrunic kidney disense; CPR-Index, C-peptide inmunoreactivity index; CVD, cardiovasculir diseases; CV-RR, coefficient of variation of R-R interval; DR, diabetic rerincpathy; $\mathrm{eGFR}$, es timate glomerular filtration rate; ECG, electrocardiogram; GA, glycated alluminin; HOMA-IR, homeostasis model assessment of ins unin resistance; MODY, maturity onset diabetes of the young; NPDR, nonproliferative diabetic retinopathy; PDR, proliferative diabetic retinopathy; PWV, pulse wave velocity; UCG, ultras onic echocartiog raphy

Figure 1. NCGM Kohnodai Diabetes Evaluation Sheet 